\title{
Modeling the Novel Coronavirus (SARS-CoV-2) outbreak in Sicily, Italy
}

\author{
Andrea Maugeri 1, Martina Barchitta ${ }^{1}$, Sebastiano Battiato ${ }^{2}$ and Antonella Agodi 1,* \\ 1 Department of Medical and Surgical Sciences and Advanced Technologies "GF Ingrassia”, University of \\ Catania, Catania, Italy \\ 2 Department of Mathematics and Computer Science, University of Catania, Catania, Italy \\ * Correspondence: agodia@unict.it
}

\begin{abstract}
Italy was the first country in Europe which imposed control measures of travel restrictions, quarantine and contact precautions to tackle the epidemic spread of the novel coronavirus (SARSCoV-2) in all its regions. While such efforts are still ongoing, uncertainties regarding SARS-CoV-2 transmissibility and ascertainment of cases make it difficult to evaluate the effectiveness of restrictions. Here, we employed a Susceptible-Exposed-Infectious-Recovered-Dead (SEIRD) model to assess SARS-CoV-2 transmission dynamics, working on the number of reported patients in intensive care unit (ICU) and deaths in Sicily (Italy), from 24 February to 13 April. Overall, we obtained a good fit between estimated and reported data, with a small fraction of unreported SARSCoV-2 cases (19.5\%; 95\%CI $=0 \%-34.7 \%$ ) before 10 March lockdown. Interestingly, we estimated that the first set of restrictions reduced transmission rate in the community by $42 \%$ (95\%CI $=38 \%-46 \%$ ), and that more stringent measures adopted on 23 March succeeded to drastically curb the transmission rate by $84 \%$ ( $95 \% \mathrm{CI}=80 \%-88 \%)$. Thus, our estimates delineated the characteristics of SARS-CoV2 epidemic before restrictions taking into account unreported data. Further modeling after the adoption of control measures, moreover, indicated that restrictions reduced SARS-CoV2 transmission considerably.
\end{abstract}

Keywords: Novel Coronavirus, COVID-19, Epidemic Model, Epidemiology

\section{Introduction}

The novel coronavirus (SARS-CoV-2) that emerged in Wuhan (China) at the end of 2019 quickly spread to other 213 countries, as of 13 April 2020. On the same date, there were 159,516 confirmed SARS-CoV-2 cases with 20,465 deaths in Italy [1]. It is worth mentioning that Italy was the first country in Europe to put its entire population on lockdown [2]. On 10 March, the Italian Government imposed control measures to the entire country, including travel restrictions, quarantine and contact precautions [3]. On $23 \mathrm{March}$, further restrictive measures, which included the shutdown of all nonstrategic production in the whole Italian territory, have been adopted [3]. Such efforts to contain the virus are still ongoing in all the Italian regions, however, uncertainties regarding SARS-CoV-2 transmissibility and virulence make it difficult to evaluate the effectiveness of restrictions. A major source of uncertainty regards the proportion of unreported cases, a critical epidemiological characteristic that might bias the interpretation of epidemic curve. Indeed, if one looks at only confirmed data, there has been a reduction of the epidemic spread in Italy after restrictions. Yet, mounting evidence suggested that undocumented infections could greatly contribute to SARS-CoV2 spread [4-6]. Moreover, several outbreaks occurred at different times scattered throughout the Italian territory [1]. For this reason, it is necessary to evaluate the epidemic curve region by region, using data that are less prone to ascertainment bias [7], such as such as those related to hospital admission and deaths [6]. 
In this study, we employed a Susceptible-Exposed-Infectious-Recovered-Dead (SEIRD) model to assess SARS-CoV-2 transmission dynamics in Sicily (South Italy). The first aim was to estimate the unknown parameters of the model before restrictions of $10 \mathrm{March}$, also assessing the possibility of unreported cases. We further modeled the transmission after 10 March to examine the impact of control measures on SARS-CoV-2 transmission.

\section{Materials and Methods}

\subsection{Formulating the SEIRD model}

As described in a previous study [6], we modeled the epidemic curve using a susceptibleexposed-infective-remove-dead (SEIRD) model, where $S(t), E(t), I(t), R(t)$, and $D(t)$ were the number of susceptible, exposed (infected but not yet be infectious), infectious, recovered and dead individuals at time ( $\mathrm{t}$ ). However, to take into account for different transmission dynamics in the community and in hospital, we separated the infectious state into three classes: patients with very mild or no symptoms in the community $\left(I_{c o m}\right)$, those with mild clinical presentation admitted to the hospital ( $\left.I_{h o s}\right)$, and those with severe disease who required Intensive Care Unit (ICU) hospitalization (Iicu) (Figure 1).

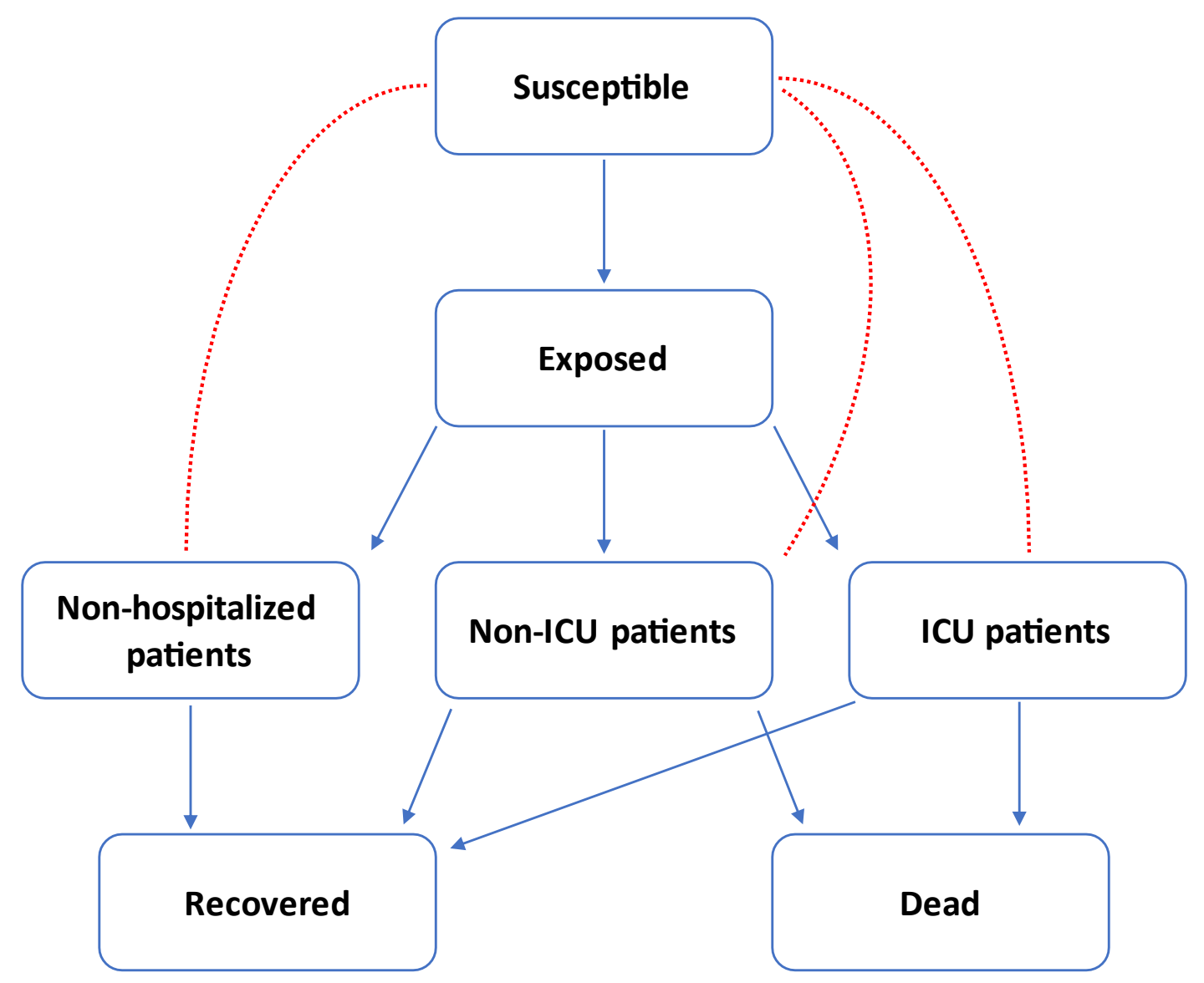

Figure 1. The employed SEIRD epidemic model for SARS-CoV-2 epidemic in Sicily, Italy.

Given that, the model was defined by the following ordinary differential equations:

1) $\frac{d S(t)}{d t}=-\frac{\left(\beta_{c o m}(t) I_{c o m}(t)+\beta_{h o s}(t) I_{h o s}(t)+\beta_{i c u}(t) I_{i c u}(t)\right) S}{N}$ 
2) $\frac{d E(t)}{d t}=\frac{\left(\beta_{c o m}(t) I_{c o m}(t)+\beta_{h o s}(t) I_{h o s}(t)+\beta_{i c u}(t) I_{i c u}(t)\right) s}{N}-\sigma E(t)$

3) $\frac{d I(t)}{d t}=\sigma E(t) v_{c o m}+\sigma E(t) v_{h o s}+\sigma E(t) v_{i c u}-\gamma I(t)$

4) $\frac{d R(t)}{d t}=\gamma_{c o m}\left(1-\mu_{c o m}\right) I_{c o m}(t)+\gamma_{h o s}\left(1-\mu_{h o s}\right) I_{h o s}(t)+\gamma_{h o s}\left(1-\mu_{i c u}\right) I_{i c u}(t)$

5) $\frac{d D(t)}{d t}=\gamma_{\text {com }} \mu_{\text {com }} I(t)+\gamma_{\text {hos }} \mu_{\text {hos }} I(t)+\gamma_{\text {hos }} \mu_{\text {icu }} I(t)$

Where:

- $\quad N$ was the total population given by the sum of each state;

- $\beta_{c o m}, \beta_{h o s}$ and $\beta_{i c u}$ were the transmission rates in the three infectious categories;

- $\sigma$ was the infection rate (i.e. the inverse of the incubation period) assumed to be the same for each infectious category;

- $\quad v_{c o m}, v_{h o s}$, and $v_{\text {icu }}$ were the probabilities that each exposed individual progressed to $I_{c o m}$, Ihos or Iicu;

- $\gamma_{\text {com }}$ and $\gamma_{\text {hos }}$ were the removing rates (i.e. assumed to be the inverse of the period between the onset of symptoms and recovering/death);

- $\quad \mu_{c o m}, \mu h o s$, and $\mu_{i c u}$ were the probabilities of dying among infectious individuals.

\subsection{Fitting the model to the reported number of ICU patients and deaths}

The SEIRD model was formulated to estimate the unknown parameters and to reflect the epidemic curve in a way consistent with reported data. However, as discussed in our previous work [6], stringent testing strategies that have been adopted in Italy and other countries might have caused an underestimation of infectious individuals, especially those with mild or no symptoms. To address the uncertainty on reported SARS-CoV-2 infections, we fitted our model to reported data on patients admitted to ICU and those who died, which were certainly less prone to be affected by ascertainment bias [7]. These data were obtained from the Italy's Civil Protection of the Italian Ministry of Health from 24 February to 13 April, 2020 [1]. In particular, we first fitted our model to the following objective function $F$ :

6) $F=E S S_{\text {icu }}+E S S_{\text {deaths }}$

Where:

- ESSicu was the error sum square of estimated and reported ICU patients from 24 February to 17 March;

- $E S S_{\text {deaths }}$ was the error sum square of estimated and reported deaths from 24 February 24 to 24 March.

In fact, given the lag of 1-2 weeks between the adoption of restrictions (i.e. first restrictions were adopted on $10 \mathrm{March}$ ) and their impact on infection and death trends, we were confident in using the above-mentioned periods to fit our model. To estimate the unknown parameters, we employed a sequential quadratic programming (SQP) algorithm for large-scale constrained optimization [8]. This algorithm has already been used for modeling purposes in the context of SARS-CoV-2 epidemic [9]. Accordingly, the following set of assumptions and constraints was defined based on the current knowledge on SARS-CoV-2 features and epidemic curve in Sicily: 
- $\quad N$ was assumed to be 5,000,000 (i.e. approximated to the current Sicilian population);

- $\quad R$ and $D$ were initially set to 0 ;

- The start date of the modeling period (i.e., February 20) corresponded 5 days (i.e. the incubation period according to Li et al. [10]) before the first case was announced in Sicily on 25 February;

- $\quad \beta_{\text {com, }} \beta_{\text {hos, }}$ and $\beta_{\text {icu }}$ were assumed to be $\geq 0.1$ and $\leq 1$;

- $\quad v_{c o m}, v_{h o s}$, and $v_{i c u}$ were assumed to be $>0.1$ and $<1$, and their sum must be 1 ;

- $\sigma$ was assumed to be $1 / 5.2$ days according to Li et al. [10];

- $\gamma_{\text {com }}$ was assumed to be $1 / 3.5$ days according to Li et al. [10], while $\gamma_{\text {hos }}$ was assumed to be $1 / 18$ days according to Wang et al. [5];

- $\quad \mu$ com was assumed to be 0 ;

- $\quad \mu$ hos was assumed to be lower than $\mu_{i c u}$.

The above process was iterated 1,000 times on randomly generated samples from the distribution functions of reported ICU patients and deaths to obtain $95 \%$ confidence intervals (CIs).

\subsection{Modeling the effect of control measures}

We further modeled the transmission of SARS-CoV-2 in Sicily after 10 March, when the control measures of travel restrictions, self-quarantine and contact precautions have been advocated by the Italian Government. To do that, we re-estimated the transmission rates in the three infectious categories (i.e., $\beta_{c o m}, \beta_{h o s}$, and $\left.\beta_{i c u}\right)$ maintaining unchanged the other parameters. In this case, we fitted the model to reported ICU patients from 18 to 27 March and reported deaths from 25 March to April 2. We used a similar approach to take into account for more stringent restrictions adopted by the Italian Government on 23 March, by fitting the model to reported ICU patients from 28 March to 13 April and reported deaths from 3 to 13 April.

\section{Results}

\subsection{Description of reported data}

Data on reported SARS-CoV-2 cases, hospitalized patients and deaths in Sicily from 24 February to 13 April are shown in Figure 2. As of 13 April 2020, there were 2,458 cases, 605 hospitalized patients and 171 deaths. Notably, the daily increment in the number of new cases decreased from 28 March to 13 April. Examining the epidemic trend, we also observed that case fatality risk - the number of deaths divided by number of cases - increased from $1.7 \%$ to $7.0 \%$ as the epidemic spread (Figure 2A). After an exponential growth, instead, the daily number of patients hospitalized in ICU or other wards reached a steady state from 28 March to 2 April followed by a slight decrement until 13 April (Figure 2B). 


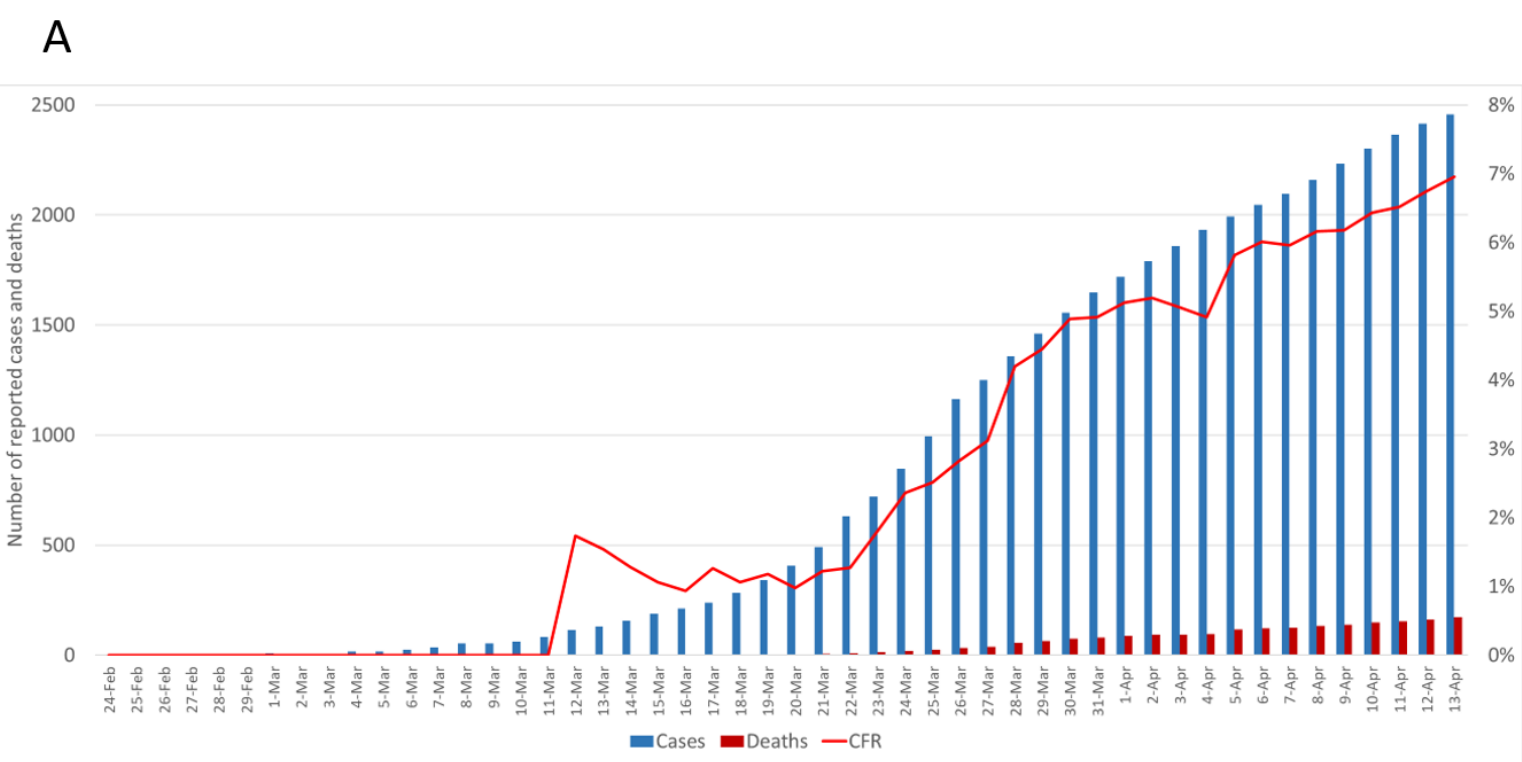

\section{B}

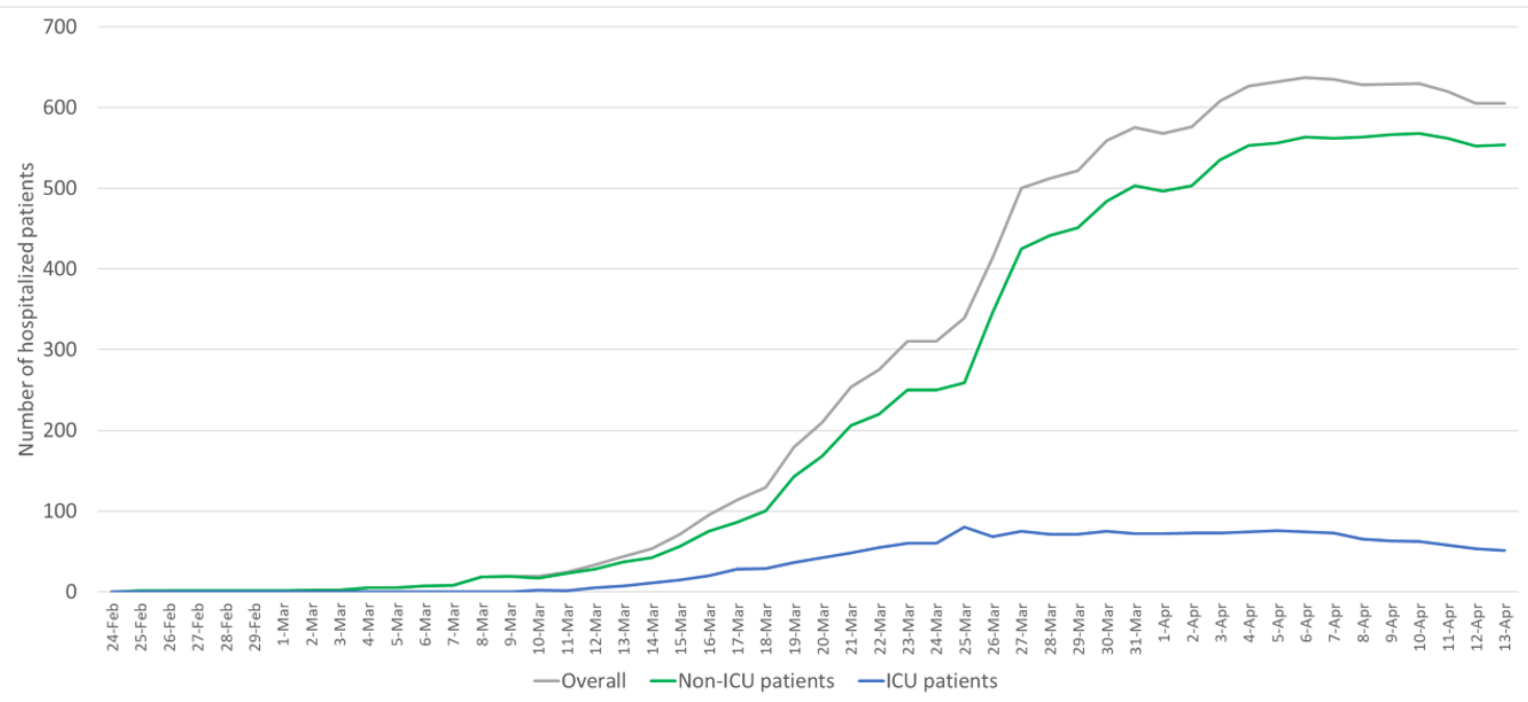

Figure 2. Number of reported cases, hospitalized patients and deaths in Sicily, Italy, from 24 February 2020 to 13 April 2020. (A) The bars represent the cumulative number of reported SARS-CoV-2 cases and related deaths while the red line represents the case fatality risk. (B)The grey line represents the daily number of hospitalized patients, while blue and green lines represent the number of those hospitalized in Intensive Care Unit (ICU) or in other wards.

\subsection{The epidemic curve prior to restrictions}

Given the uncertainty on reported data, especially those related to confirmed cases, we first fitted the SEIRD model to reported ICU patients and deaths, obtaining an overall good fit between estimated and reported data (Figure 3A). The estimated parameters which best explained the reported numbers of ICU patients and deaths are shown in Table 1. Using the best-fitting parameters, we estimated a total of 77 (95\%CI=61-95) SARS-CoV-2 cases on 10 March, which comprised 62 $(95 \% \mathrm{CI}=49-77)$ infectious individuals, $30(95 \% \mathrm{CI}=23-37)$ hospitalized patients (4 patients in ICU; $95 \% \mathrm{CI}=3-5$ and 26 patients in other wards; $95 \% \mathrm{CI}=20-32), 14$ recovered (95\%CI=11-17), and 1 
(95\%CI=0-1) deaths. Although these estimates revealed only a small proportion of unreported cases $(19.5 \% ; 95 \% \mathrm{CI}=0 \%-34.7 \%)$, the estimated case fatality risk of $3.5 \%$ would remain stable as the epidemic spread. It was also noteworthy that, in absence of restrictions, these estimates would reach 10727 (95\%CI=6,022-18,386) patients hospitalized in ICU and 1,683 (95\%CI=986-2792) deaths on 13 April (Figure 3B).

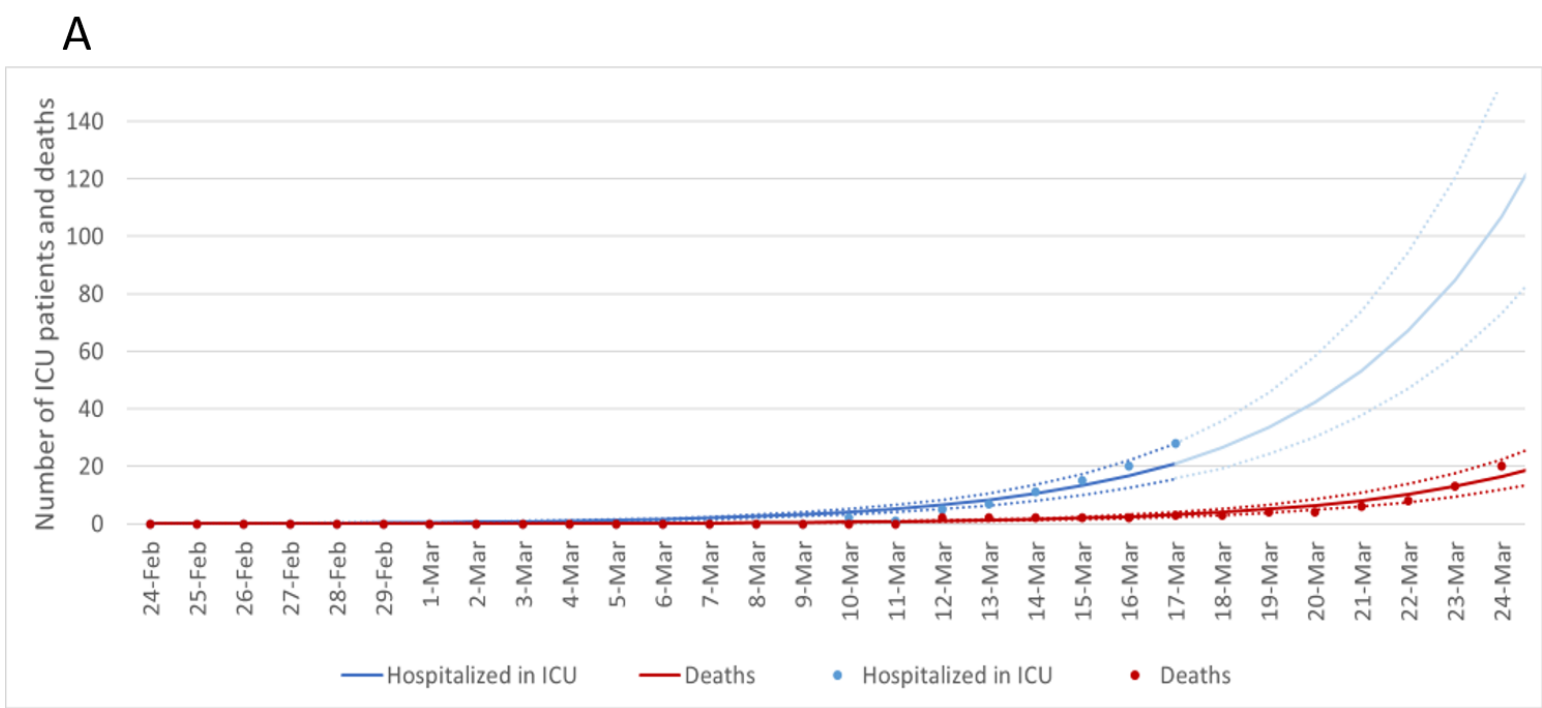

B

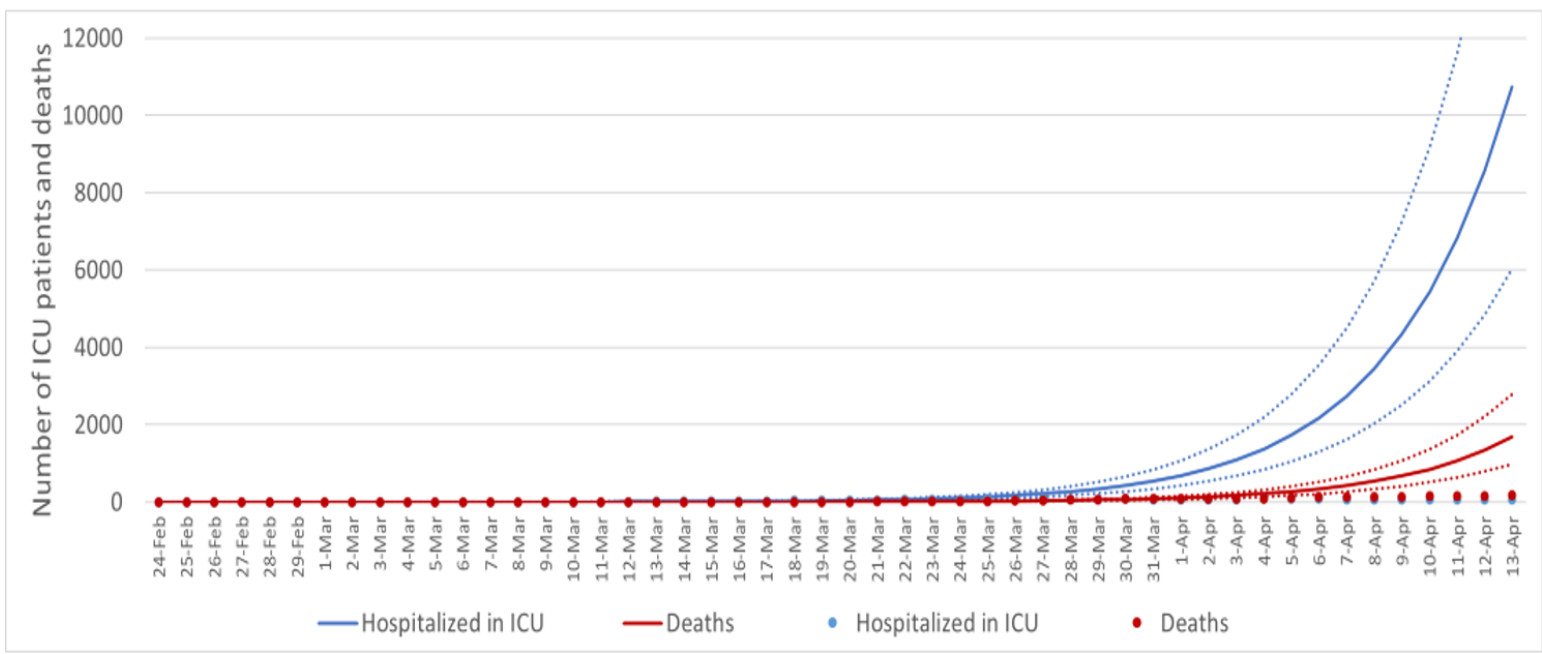

Figure 3. Number of estimated and reported patients in Intensive Care Unit and deaths in Sicily, Italy, in absence of restrictions. (A) Fitting the SEIRD model to the reported number of patients admitted to Intensive Care Unit (ICU) and deaths without restrictions. (B) Extension of the model without restrictions until 13 April 2020. The dots represent the number of reported ICU patients and deaths while the lines represent the estimate and 95\% confidence intervals through the SEIRD model, along the temporal axis. 
Table 1. Best-fitting parameters in absence of control measures

\begin{tabular}{|c|c|c|}
\hline SEIRD Parameters & Definition & Estimated values $(95 \% \mathrm{CI})$ \\
\hline$\beta_{\text {com }}$ & \multirow{3}{*}{ Transmission rate } & $0.89(95 \% \mathrm{CI}=0.88-0.90)$ \\
\hline$\beta_{\text {hos }}$ & & $0.48(95 \% \mathrm{CI}=0.47-0.49)$ \\
\hline$\beta_{i c u}$ & & $0.36(95 \% \mathrm{CI}=0.34-0.38)$ \\
\hline $\boldsymbol{v}_{\text {com }}$ & \multirow{3}{*}{$\begin{array}{l}\text { Probability of progression to Icom, } \\
\qquad I_{h o s, ~} \text { or } I_{i c u}\end{array}$} & $0.52(95 \% \mathrm{CI}=0.50-0.54)$ \\
\hline Vhos & & $0.40(95 \% \mathrm{CI}=0.38-0.42)$ \\
\hline$v_{\text {icu }}$ & & $0.08(95 \% \mathrm{CI}=0.06-0.10)$ \\
\hline$\mu_{\text {hos }}$ & \multirow{2}{*}{ Probability of dying } & $0.05(95 \% \mathrm{CI}=0.02-0.08)$ \\
\hline$\mu_{i c u}$ & & $0.68(95 \% \mathrm{CI}=0.66-0.72)$ \\
\hline
\end{tabular}

\subsection{The effect of restrictions on transmission rates}

We next applied the SEIRD model to evaluate the effect of restrictions adopted by the Italian Government on 10 March and then tightened on 23 March (Figure 4). We estimated that the first set of restrictions reduced transmission rate in the community by $42 \%$ ( $95 \% \mathrm{CI}=38 \%-46 \%)$. The restrictions, actually, were also able to slightly reduce transmission rates of patients hospitalized in ICU or in other wards by $18 \%(95 \% \mathrm{CI}=15 \%-21 \%)$ and $11 \%(95 \% \mathrm{CI}=9 \%-13 \%)$, respectively. However, only more stringent measures adopted on 23 March succeeded to drastically curb the transmission rate in the community by $84 \%(95 \% \mathrm{CI}=80 \%-88 \%)$, maintaining almost unaltered the transmission dynamics in hospital.

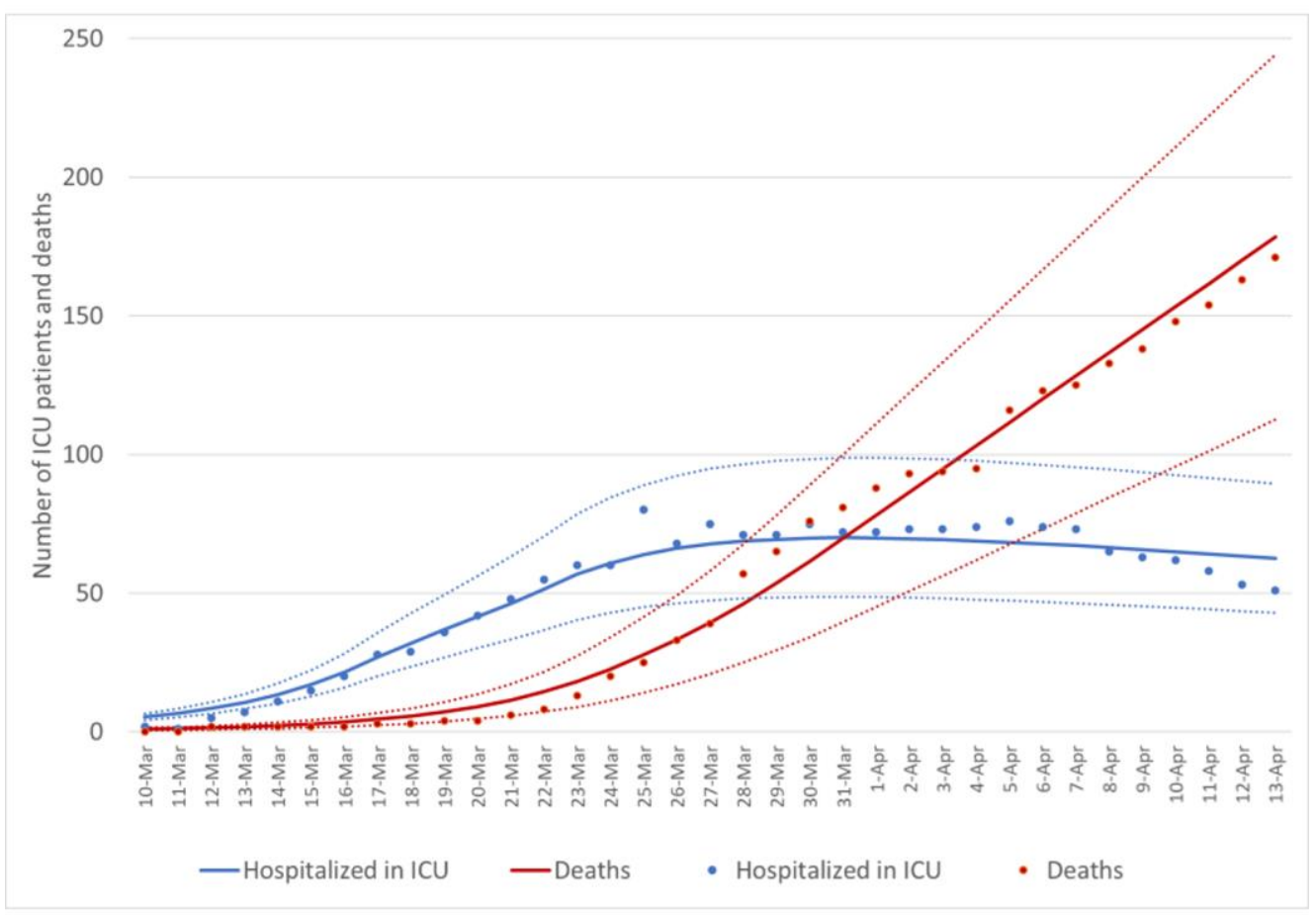

Figure 4. Modeling of control measures adopted on 10 March 2020 and then tightened on 23 March 2020. The dots represent the number of reported ICU patients and deaths while the lines represent the estimate and 95\% confidence intervals through the SEIRD model, along the temporal axis. 


\section{Discussion}

In this study, we modeled the SARS-CoV-2 epidemic in Sicily (South Italy) to estimate any instances of undocumented infections and to evaluate the impact of control measures adopted by the Italian Government. Our approach stemmed by the hypothesis that looking at reported cases could lead to biased speculations when interpreting data on an epidemic emergency. For this reason, we further adapted a our previously described SEIRD model [6] to investigate the SARS-CoV-2 epidemic curve in Sicily, working directly on data on admissions to ICU and deaths. A similar hypothesis has been formulated by a research group from the Imperial College of London, who is investigating the epidemic curve on the basis of deaths observed over time [11]. However, to our knowledge, our study was the first employing also data on admissions to ICU, another event that was certainly less affected by reporting bias. Our findings revealed a smaller proportion of unreported cases in Sicily if compared with previous estimates in Italy [12]. A plausible explanation was that the Sicily's regional health system did not experience the same emergency observed in other Italian regions, such as Lombardy, Emilia Romagna, and Piedmont [13]. As such, despite stringent testing strategies, only $\sim 20 \%$ of cases were not documented on 10 March. However, the small proportion of unreported cases biased the apparent increase in case fatality risk during the epidemic spread. Instead, taking into account estimated cases, case fatality risk stably stood around 3.5\%. As stated by previous studies [13], indeed, stringent testing strategies adopted in Italy might explain the higher case fatality risk than other countries. This raised the need for caution when comparing preliminary estimates between and within countries.

If we extended our model without accounting for restrictions, our estimates depicted a dramatic scenario, with more than 10,000 patients hospitalized in ICU and 1,600 deaths on 13 April. In absence of control measures, this would have constituted a serious threat for the Sicily's regional health system. Nevertheless, restrictive measures imposed by the Italian Government in the middle of March succeeded in containing the epidemic spread. Measures such as travel restrictions, quarantine and contact precautions in the community modified the transmission rate of SARS-CoV-2. Interestingly, the transmission rate in the community decreased by $42 \%$ after 10 March and by $84 \%$ after 23 March. Beside this, changes in healthcare worker's behavior such as wearing of facemasks, social distancing, self-isolation when sick contributed by reducing transmission rates in the hospital setting. Indeed, our estimates on the impact of control measures were in line with those reported by $\mathrm{Li}$ and colleagues on the Chinese experience [4].

We recognized these findings were based on an epidemic model and that therefore required caution. However, we fitted our model to those data that were considered less biased by uncertainty such as admissions to ICU and deaths. Indeed, in a region where ICU bed needs for SARS-CoV-2 had not yet reached saturation, even the reported number of patients in ICU could be considered less biased. With the considerations in mind, our estimates offered potential explanations for the current understanding of SARS-CoV-2 epidemic in Sicily. Our approach certainly relied on several assumptions and constraints based on the current knowledge on SARS-CoV-2 characteristics and relevant references published in the last months. Despite this, however, we cannot rule out some degree of uncertainty of our estimates.

In conclusion, our approach - which assessed the epidemic curve by estimating backwards from the documented admissions to ICU and deaths - appeared to be useful for the investigation of SARSCoV2 epidemic. Our model on the early phase of the outbreak delineated the characteristics of SARS$\mathrm{CoV} 2$ transmission in a region without major restrictions or control. Further analysis extended after the adoption of control measures, instead, indicated that restrictions reduced SARS-CoV2 transmission considerably. Thus, similar strategies would have to be implemented outside Italian regions to control the epidemic spread. 
Author Contributions: Conceptualization, A.M. and A.A.; methodology, A.M. and S.B.; software, A.M.; formal analysis, A.M. and M.B.; data curation, A.M. and S.B.; writing-original draft preparation, A.M. and M.B.; writing-review and editing, all the Authors; visualization, A.M.; supervision, A.A. All authors have read and agreed to the published version of the manuscript.

Funding: This research was funded by the Assessorato della Salute, Regione Siciliana - Progetti Obiettivo di Piano Sanitario Nazionale.

Conflicts of Interest: The authors declare no conflict of interest.

\section{References}

1. Italian Ministry of Health. Covid-19. Situation report update at 13 April 18:00. Availabe online: http://www.salute.gov.it/portale/nuovocoronavirus/homeNuovoCoronavirus.jsp?lingua=english (accessed on April 14, 2020).

2. World Health Organization. Health topics: Coronavirus. Availabe online: https://www.who.int/healthtopics/coronavirus\#tab=tab_1 (accessed on April 14, 2020).

3. Italian Ministry of Health. Novel coronavirus. Availabe online: http://www.salute.gov.it/portale/nuovocoronavirus/homeNuovoCoronavirus.jsp?lingua=english (accessed on 14 April, 2020).

4. Li, R.; Pei, S.; Chen, B.; Song, Y.; Zhang, T.; Yang, W.; Shaman, J. Substantial undocumented infection facilitates the rapid dissemination of novel coronavirus (SARS-CoV2). Science 2020, doi:10.1126/science.abb3221.

5. Wang, H.; Wang, Z.; Dong, Y.; Chang, R.; Xu, C.; Yu, X.; Zhang, S.; Tsamlag, L.; Shang, M.; Huang, J., et al. Phase-adjusted estimation of the number of Coronavirus Disease 2019 cases in Wuhan, China. Cell Discov 2020, 6, 10, doi:10.1038/s41421-020-0148-0.

6. Maugeri, A.; Barchitta, M.; Battiato, S.; Agodi, A. Estimation of Unreported Novel Coronavirus (SARS-CoV2) Infections from Reported Deaths: a Susceptible Exposed Infectious Recovered Dead Model. Preprints 2020, doi:10.20944/preprints202004.0052.v1.

7. Lipsitch, M.; Donnelly, C.A.; Fraser, C.; Blake, I.M.; Cori, A.; Dorigatti, I.; Ferguson, N.M.; Garske, T.; Mills, H.L.; Riley, S., et al. Potential Biases in Estimating Absolute and Relative Case-Fatality Risks during Outbreaks. PLoS Negl Trop Dis 2015, 9, e0003846, doi:10.1371/journal.pntd.0003846.

8. Gill, P.E.; Murray, W.; Saunders, M.A. Large-scale SQP Methods and their Application in Trajectory Optimization. In Computational Optimal Control, Bulirsch, R., Kraft, D., Eds. Birkhäuser Basel: Basel, 1994; pp. 29-42.

9. Zhu, H.; Li, Y.; Jin, X.; Huang, J.; Liu, X.; Qian, Y.; Tan, J. Transmission Dynamics and Control Methodology of COVID-19: a Modeling Study. medRxiv 2020, doi:https://doi.org/10.1101/2020.03.29.20047118.

10. Li, Q.; Guan, X.; Wu, P.; Wang, X.; Zhou, L.; Tong, Y.; Ren, R.; Leung, K.S.M.; Lau, E.H.Y.; Wong, J.Y., et al. Early Transmission Dynamics in Wuhan, China, of Novel Coronavirus-Infected Pneumonia. N Engl J Med 2020, 382, 1199-1207, doi:10.1056/NEJMoa2001316.

11. Imperial College COVID-19 Response Team. Estimating the number of infections and the impact of nonpharmaceutical interventions on COVID-19 in 11 European countries; 2020.

12. Tuite, A.R.; Ng, V.; Rees, E.; Fisman, D. Estimation of COVID-19 outbreak size in Italy. Lancet Infect Dis 2020, doi:10.1016/S1473-3099(20)30227-9.

13. Onder, G.; Rezza, G.; Brusaferro, S. Case-Fatality Rate and Characteristics of Patients Dying in Relation to COVID-19 in Italy. JAMA 2020, doi:10.1001/jama.2020.4683. 